Molecular-dynamics investigation on polarization retention of barium titanate nanofilm arising from ordered oxygen vacancy

This article has been downloaded from IOPscience. Please scroll down to see the full text article.

2010 EPL 9217006

(http://iopscience.iop.org/0295-5075/92/1/17006)

View the table of contents for this issue, or go to the journal homepage for more

Download details:

IP Address: 159.226.100.225

The article was downloaded on 23/03/2011 at 10:11

Please note that terms and conditions apply. 


\title{
Molecular-dynamics investigation on polarization retention of barium titanate nanofilm arising from ordered oxygen vacancy
}

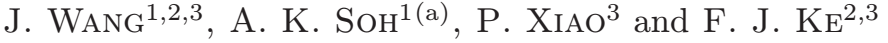 \\ ${ }^{1}$ Department of Mechanical Engineering, The University of Hong Kong - Hong Kong, PRC \\ ${ }^{2}$ School of Physics and Nuclear Energy Engineering, Beihang University - Beijing 100191, PRC \\ ${ }^{3}$ State Key Laboratory of Nonlinear Mechanics (LNM), Institute of Mechanics, \\ Chinese Academy of Sciences - Beijing 100190, PRC
}

received 25 May 2010; accepted in final form 22 September 2010

published online 29 October 2010

PACS 77.80.Dj - Domain structure; hysteresis

PACS 77.55.fe- $\mathrm{BaTiO}_{3}$-based films

\begin{abstract}
Molecular-dynamics simulations have been carried out to investigate the electric hysteresis of barium titanate nanofilm containing oxygen vacancy ordering array parallel to the $\{101\}$ crystal plane. The results obtained show a significant weakening of polarization retention from non-zero value to zero as the size of the array was reduced to a critical level, which was attributed to the formation and motion of head-to-head domain wall structure under external field loading process. By comparing with materials containing isolated oxygen vacancies, it was found that the zero retention was due to the oxygen vacancy ordering array rather than to the concentration of oxygen vacancy.
\end{abstract}

Copyright (C) EPLA, 2010

Ferroelectric barium titanate (BTO) is widely used to fabricate memories [1,2], sensors [3], actuators [4] and other integrated miniature devices due to its outstanding properties, which include ferroelectricity, piezoelectricity, pyroelectricity, large dielectric constants, etc. In particular, hysteresis is one of the most significant ferroelectric behaviors, and the fundamental properties, such as retention $[1,5]$, coercive force and saturated field, are essential characteristics to be taken into consideration for using BTO to make memories. Retention is an important property because a BTO with weak retention cannot be used to make data recording, and devices are completely inoperable if the retention is reduced to zero. In the last decade, many researchers have been attracted to study the weakening of retention of BTO resulting from oxygen vacancy. To-date, although some qualitatively different models have been proposed, such as concentration [6], oxygen vacancy ordering [7], electromigration of oxygen vacancies to form extended defects capable of pinning domains [8], etc., the microscopic aspect of the weakening, i.e., the effect of vacancy structures on retention, remains unclear. Therefore, the present study focuses on weakening of retention of (001) $\mathrm{TiO}_{2}$-terminated BTO nanofilms of $10 \mathrm{~nm}$ thickness under the conditions of i) no oxygen vacancy, ii) vacancies in ordering array, iii) isolated ordered vacancies, and

(a) E-mail: aksoh@hku.hk iv) isolated vacancies. The proposed study is devised based on the following considerations: i) the behavior of BTO in memories is closer to that of film than bulk; ii) the images of transmission electron microscopy (TEM) [9] have shown that the vacancies can form an ordering structure parallel to $(1 \overline{1} 0)$ and the distance between two neighboring chains is about 5 cells; and iii) it has been found that the oxygen vacancy chain along [101] and parallel to (101) [8] would lead to fatigue of BTO. Since fatigue renders degradation of ferroelectric behavior with reduction of polarization, coercive force, etc., if the proposed structure can lead to fatigue, it may also cause weakening of retention. Furthermore, there is a lack of literature with regards to the dependence of ferroelectric behavior on the dimension of the vacancy structure. Therefore, there is a need to carry out research to seek quantitative description and microscopic view in this aspect.

The $\mathrm{TiO}_{2}$-terminated film model devised contains $n \times$ $m \times 25.5$ perovskite cells, where $n$ and $m$ are integers used as auxiliary variables to adjust the dimension of the oxygen vacancy array. Note that the minimum value of $n$ and $m$ used in the present work is 6 , which is determined based on the cutoff distance of $1.0 \mathrm{~nm}$ and the perovskite cell constant of $0.4 \mathrm{~nm}$. The cutoff distance of $1.0 \mathrm{~nm}$ is adequate to repeat the phase sequence [10] and there is no significant discrepancy by increasing it to $1.1 \mathrm{~nm}$. The comparison of the out-of-plane polarization 


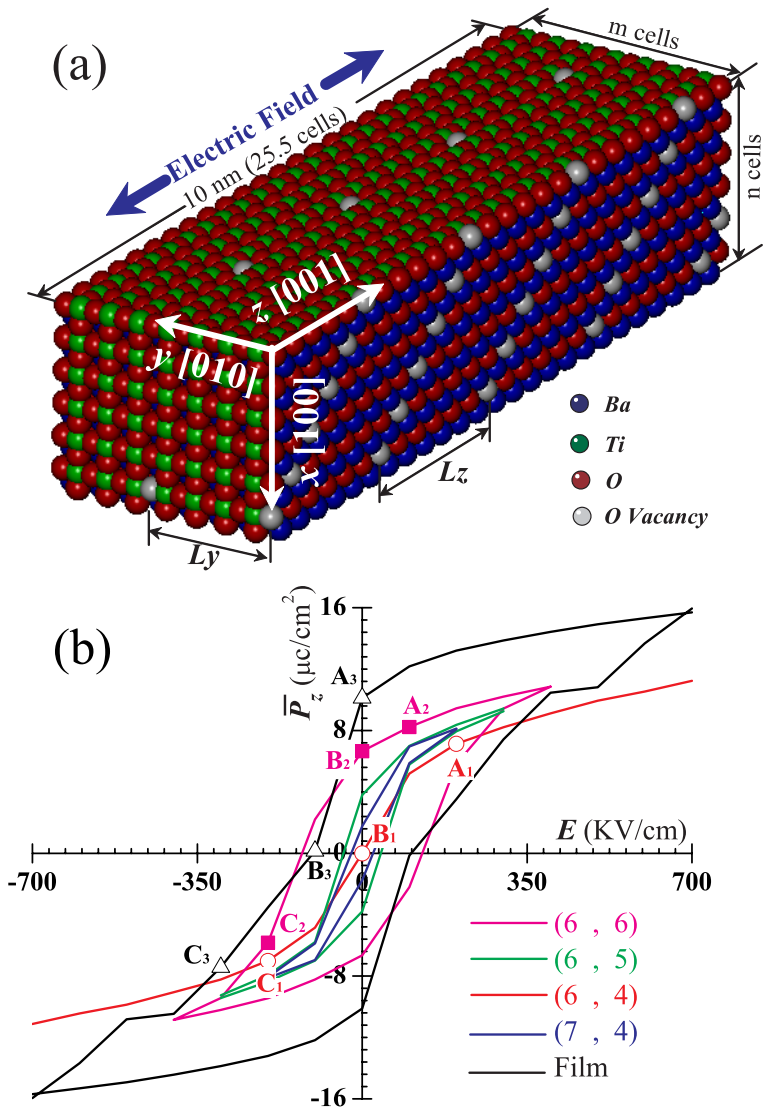

Fig. 1: (Color online) (a) Pattern of the $(6,4)$ array and (b) hysteresis loops.

patterns obtained from in-plane film models of $12 \times 12$ and $6 \times 6$ cells shows that the two sets of results are in agreement in the sense that both show half the polarization points in the [001] direction and the other half points in the opposite direction, and the smallest size is 2 cells; moreover, the biggest dimension is infinite due to the periodic boundary conditions. This means that the in-plane size of $6 \times 6$ cells is adequate for the prediction of domain sizes. Figure 1 (a) shows a $6 \times 8 \times 25.5$ system. The $x, y$ and $z$ axes coincide with [100], [010] and [001] orientations, respectively. A vacuum region of $2 \mathrm{~nm}$ is attached on the outside of the two $\mathrm{TiO}_{2}$-terminated ends (along $z$ ) and periodic boundary conditions are implemented along all the three directions to simulate a film of $10 \mathrm{~nm}$ thickness. Tinte and Stachiotti [11], who proposed the core-shell model, had proven that the size of $2.0 \mathrm{~nm}$ was adequate to minimize the interaction between periodic images [11]. Oxygen vacancies are generated by removing the oxygen atoms and their corresponding charges directly. The vacancy ordering array is parallel to the $\{101\}$ crystal plane and the dimension of this array is depicted by two parameters. One is the separation between two ordering along the $z$-direction $(L z)$ and the other is that along the $y$-direction $(L y)$, which can divide $n$ and $m$ into equal parts, respectively. The vacancy ordering array is generated by the following steps: i) generation of an ordering line on the (101) plane which is perpendicular to the $[010]$ orientation; and ii) repetition of such ordering line along [001] and [010] orientations at intervals of $L z$ and $L y$ measured on perovskite cells, respectively. Moreover, isolated vacancies are generated uniformly in a $6 \times 6 \times 25.5$ cells system.

For the molecular-dynamics (MD) framework, an isotropic core-shell model [10] developed from first principle [12] is employed in the present study to depict the interactions among ions. The said model has been successfully employed to depict the phase transition sequence that depends on temperature [10], size dependence of film [13] and wire [14], surface properties and influence of epitaxial strain on ferroelectric behavior [11] and, most importantly, the point-defect formation energy [6], which is significant in a point-defect system. The potential parameters are taken from ref. [15], and the approach used in ref. [13], in which the charge on the titanate was taken as that at neutral condition, is adopted, i.e., the extra charge is uniformly redistributed on $\mathrm{Ti}$ cores in order to keep the system neutral. An adiabatic dynamics is used to integrate the motion of shells by assigning small masses to the shells [16]; long-ranged coulomb interactions are calculated by Wolf sum [17]; the applied external field along the $z$-direction is in the range of $-1500 \mathrm{kV} / \mathrm{cm}$ to $1500 \mathrm{kV} / \mathrm{cm}$, and the increment is $100 \mathrm{kV} / \mathrm{cm}$ for loading and $-100 \mathrm{kV} / \mathrm{cm}$ for inverse loading. The imposition of field and calculation of polarization are the same as those carried out in refs. [13,14]. All calculations are carried out using the DL_POLY package [18], which employs the Berendsen constant $(N, \sigma, T)$ algorithm with 8 Ba cores fixed, 4 on each surface, which are uniformly distributed on the surfaces. This setting allows fluctuation of all cell angles and lengths and eliminates reduction of the vacuum region. In the present study, the temperature is set as $10 \mathrm{~K}$ to minimize thermal effect and ensure excellent ferroelectric behavior. Moreover, the pressure is set as zero to ensure no involvement of stress/strain effects. The total time taken for applying a field is $12 \mathrm{ps}$ with a time step of $0.4 \mathrm{fs}$, and statistical values are extracted from the last $6 \mathrm{ps}$, which is adequate to provide sufficient accuracy for the integration of the shell coordinates as well as precision for statistical values.

In dealing with vacancy ordering array, firstly, $L z$ is fixed at 6 cells and $L y$ is varied in the range of 6 to 4 cells (denoted as $(6,6),(6,5),(6,4)$ array, etc.). Based on the above discussion, the supercells used for the study of $(6,4)$, $(7,4)$ and $(6,5)$ arrays are $6 \times 8 \times 25.5,7 \times 8 \times 25.5$ and $6 \times 10 \times 25.5$ cells, respectively. Figure $1(\mathrm{~b})$ shows that there is an obvious weakening of retention with a reduction of residual polarization $\left(\bar{P}_{z r}\right)$ from $6.7 \pm 0.2$ to $0.0 \pm$ $0.3 \mu \mathrm{C} / \mathrm{cm}^{2}$. The coercive force $\left(E_{c}\right)$ and saturated field $\left(E_{s}\right)$ exhibit the same attenuation trend, as presented in table 1 . Subsequently, when $L y$ is fixed at 4 cells and $L z$ is increased to 7 cells, the residual polarization is increased to $1.8 \pm 0.6 \mu \mathrm{C} / \mathrm{cm}^{2}$. Obviously, zero retention, which stands for no net polarization, is exhibited when $L z$ and $L y$ are 
Table 1: Critical values of the hysteresis loop.

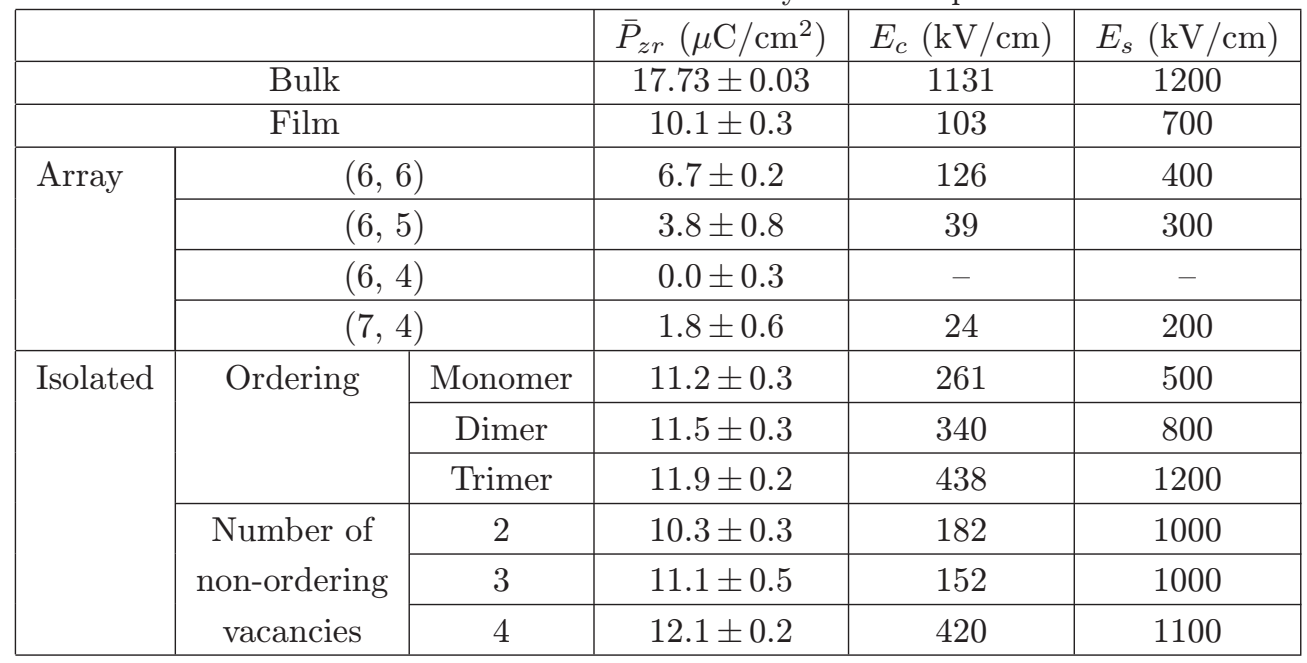

lower than or equal to 6 and 4 cells, respectively. In other words, the dimension of the $(6,4)$ array is critical for weakening of polarization retention from non-zero value to zero. It appears that zero retention would occur when the concentration of oxygen vacancy is increased, which is corresponding to a reduction of array size, to a certain level. The $(6,4)$ array corresponds to a concentration of $0.94 \%$, which is not as high as those proposed in the open literatures $[8,9]$. To ascertain the influence of oxygen vacancy concentration on the material behavior, the $(6,4)$ array is selected for refilling oxygen ions into the previous vacancies, and the number of inserted ions varies from zero to half that of the vacancies. The refilling is done by inserting one ion at an interval of several vacancies, e.g., inserting one ion in every two vacancies. Thus, the concentration can be reduced to just half that of the initial value. It is interesting to note that only zero retention is observed. This demonstrates that instead of concentration, oxygen vacancy ordering is the actual cause of zero retention for the array. The mechanism of zero retention resulting from vacancy ordering array can be attributed to the motion of a head-to-head domain wall structure [19], as illustrated in fig. 2. Due to the existence of the array, the whole system is divided into striped zones of characteristic size which is the same as that of the array. As the size of the array is decreased below a certain level, a head-to-head domain wall structure is forming as a result of spontaneous polarization, as illustrated in fig. 2(a) and (b) where local polarizations of the upper and lower ends point inward and the middle disordered region constitutes a wall. The dimension of the wall is about 4 to 5 cells. When the applied field is reduced from 200 to $0 \mathrm{kV} / \mathrm{cm}$, this structure moves from the upper end to the centre accompanying with reorientation of dipoles, as illustrated in fig. 2(c) to (h) which shows the complete process of domain wall motion. One important observation is that in the vicinity of a vacancy, local polarizations show tubular-radial distribution with [101]
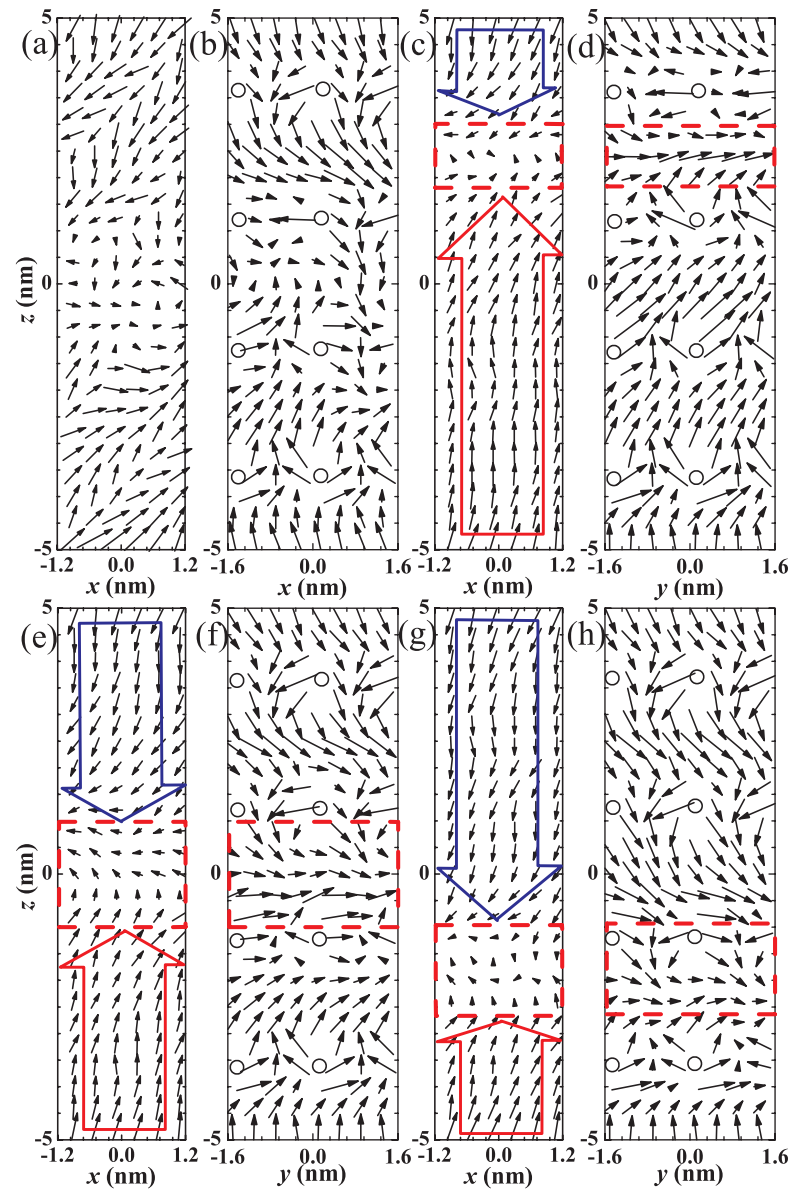

Fig. 2: (Color online) Motion of the head-to-head domain wall structures. (a) and (b) show the distribution on xoz and yoz planes before applying the electric field. (c) and (d) to (g) and (h) are patterns corresponding to an applied electric field of 200,0 and $-200 \mathrm{kV} / \mathrm{cm}$, respectively, i.e., points $\mathrm{A}_{1}$ to $\mathrm{C}_{1}$ in fig. 1(b). Domain walls are marked with dashed boxes. Blue and red hollow arrows denote upward and downward orientations of domains separated by the wall, respectively. Circles denote oxygen vacancies. 
as the axis pointing away from the vacancy chain; and the corresponding cell polarization is also bigger than that of other regions which do not contain defects, as illustrated in fig. 2(b), (d), (f) and (h). This phenomenon may be attributed to the fact that in the dipole space, a vacancy chain can generate an equivalent tubular surface. Thus, the polarization distribution near the chain is similar to the local distribution near the surfaces. It can be clearly seen from fig. 2 and the subsequent figures, which show polarization patterns, that the cell polarization near the surface points to the inner side and the magnitude is greater than that of the regions without defects. Note that Zhang et al. [14] have also found a similar surface phenomenon on BTO nanowires by $a b$ initio and MD caculations.

Since the temperature is set at $10 \mathrm{~K}$ in the present work, one may feel that the spontaneous polarization should be along the [111] direction. However, the above description seems to suggest that the structure is tetragonal with polarization along the [001] direction. To clarify this doubt, a quantitative analysis is carried out by calculating the magnitude and angle distribution of dipoles, which are presented in fig. 3. Upon accomplishment of spontaneous polarization, the magnitude of cell polarization is varied between 1.3 and $33.1 \mu \mathrm{C} / \mathrm{cm}^{2}$ with an average value of $16.9 \mu \mathrm{C} / \mathrm{cm}^{2}$. Under the assumption that the average value is the expected value, the theoretical Gaussian distribution can thus be obtained. Figure 3(a) shows that the distribution of the polarizations calculated from MD is in good agreement with the Gaussian distribution. Note that the angle distribution is defined as the ratio of the number of dipoles in a certain orientation, which is at an angle away from a reference orientation, with respect to the total dipoles in all possible orientations. In the present study, by selecting [001] as the reference orientation, the polarizations tend to align along orientations which are at $45^{\circ}$ with respect to the [001] direction, as illustrated in fig. 3(b), which shows similar polarization distributions for the case of spontaneous polarization (before an electric field is applied) and that when the electric field is reduced to 0 . The two peaks occurring at $45^{\circ}$ and $135^{\circ}$ demonstrate that two anti-parallel main domains are formed, and the width of peak indicates the extent of polarization deviation from the peak angle. Figure 3(c) also shows the shifting of peak from $45^{\circ}$ to $135^{\circ}$ by reducing the field from 200 to $-200 \mathrm{kV} / \mathrm{cm}$, which indicates the growth of one domain and the perishing of the opposite one. By carrying out a thorough analysis, the polarization is found to be parallel to [101] or the equivalent orientations, which can also be clearly seen in fig. 2(a) to (h) where most local polarizations are at $45^{\circ}$ angle in both the $x o z$ and yoz planes. This orthorhombic behavior is known as the "ac" phase in the phase diagram presented by Diéguez et al. [20], which differs from that of bulk BTO at $10 \mathrm{~K}$, maybe originated from the planar film geometry and the associated depolarization effects. In Diéguez's diagram of zero-misfit strain, the energy of the "ac" phase is slightly higher than that of the "r" phase. Since a
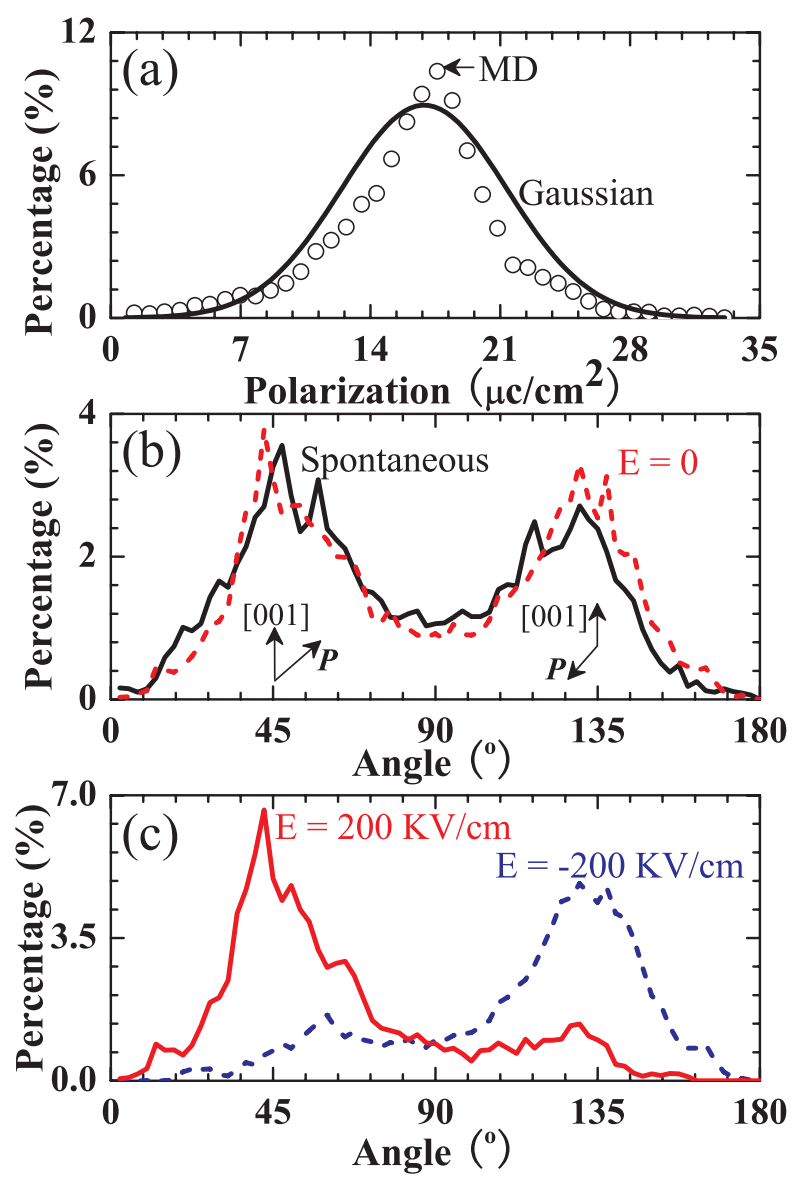

Fig. 3: (Color online) Distributions of the magnitude and angle of polarizations. (a) Magnitude distribution. Circles and solid line correspond to MD calculation data and Guassian distribution, respectively. (b) Angle distribution by selecting [001] as reference orientation. $E=0$ denotes the case when the external field is decreased to 0 , and the other curve shows spontaneous polarization. (c) Angle distribution when the external field is decreased 200 and $-200 \mathrm{kV} / \mathrm{cm}$.

free surface possesses higher energetic status, the phase transformation from " $\mathrm{r}$ " to "ac" is attainable.

However, for a dimension greater than the $(6,4)$ array, this head-to-head structure does not exist because the dominating electric behavior of domain switching is completely different. For example, the domain switching of the $(6,6)$ array is accompanied by forming and perishing of local vortex structures, as shown in fig. 4(a) to (h) which show the dipole distributions for the applied field varying from 100 to $-200 \mathrm{kV} / \mathrm{cm}$. Note that the vortex structures are located near the upper end. As the applied field is reduced from saturation to zero, there is almost no change in domain orientations except for a little change of dipole orientations near the vortex structure. By increasing the applied field in the reverse direction, the vortex structure would appear first before the occurrence of domain reorientation. When reorientation of all domains is completed, saturation is reached. Refer to fig. 4 which illustrates the complete switching process of domains. However, this behavior is not the same as that of a 


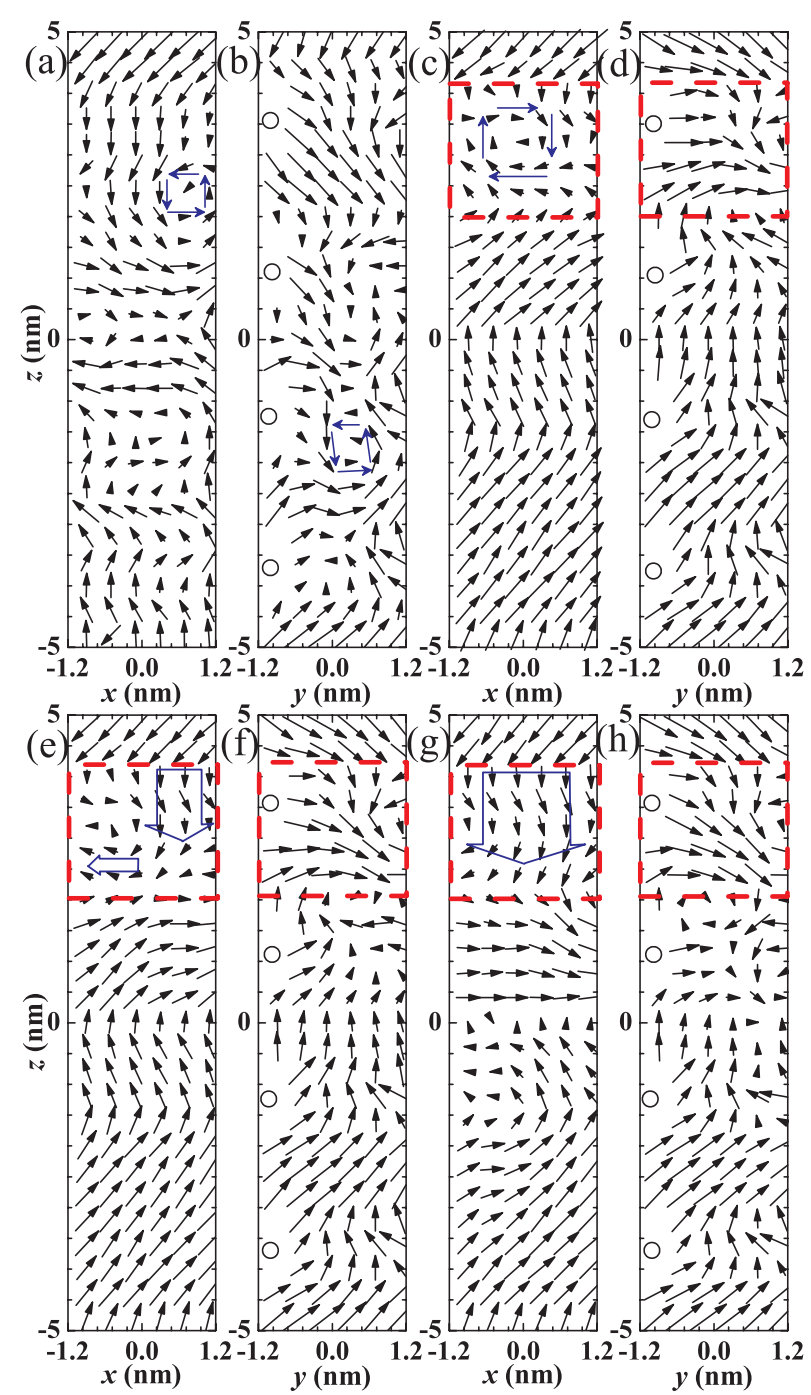

Fig. 4: (Color online) Domain switching and reorientation. (a) and (b) are spontaneous polarization patterns. (c) and (d), (e) and (f), and (g) and (h) correspond to points $\mathrm{A}_{2}$ to $\mathrm{C}_{2}$ in fig. 1(b). Vortex structures are marked by thinner arrows. The region marked with a dashed box shows the typical domain switching and reorientation mode of the $(6,6)$ array. The hollow arrows indicate the local orientation. Circles represent oxygen vacancies.

nanofilm, for which although the vortex structure is also the precursor of domain reorientation, the vortex is much clearer and it only occurs when the applied field is reduced to zero, as illustrated in fig. 5(a) to (h). The reorientation occurs in almost every domain that forms penetrating stripes from the upper to the lower surface with characteristic width of 2 to 3 cells as the reverse field is increased. And the stripe grows both in width and length by the reorientation of nearby dipoles during the loading process till reorientation is completed and saturation is reached. Refer to fig. 5 which presents the penetrating process. Note that a similar process has been reported in ref. [21]. It is worthwhile to mention that Drezner et al. have reported the formation of a 1-2 cells wide stripe domain in ultra-thin BTO films of thickness less than

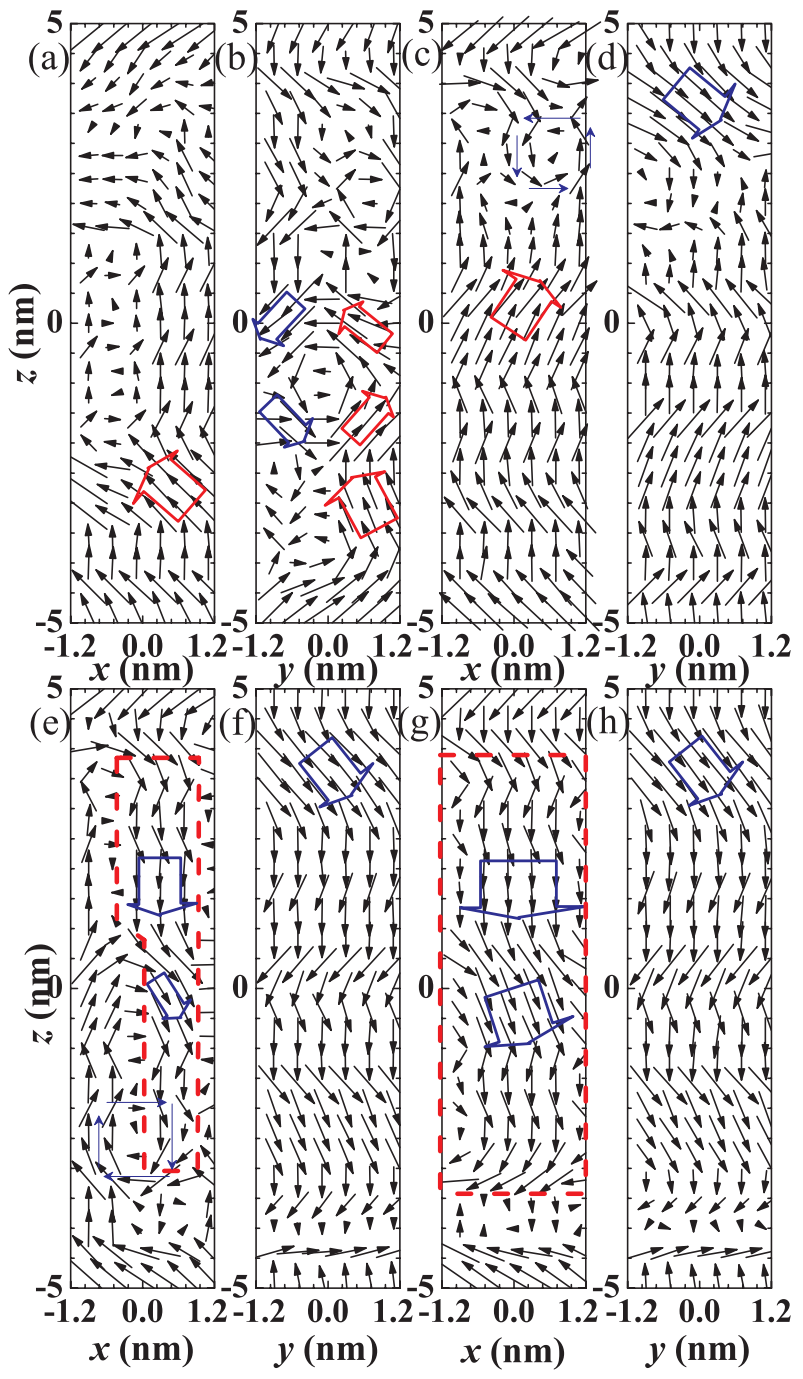

Fig. 5: (Color online) Formation and growth of the penetrating domain strip. (a) and (b) illustrate spontaneous polarization before applying the electric field. (c) and (d), (e) and (f), and $(\mathrm{g})$ and $(\mathrm{h})$ relate to points $\mathrm{A}_{3}$ to $\mathrm{C}_{3}$ in fig. $1(\mathrm{~b})$. Vortex structures are marked with thinner arrows. The dashed polygon shows the growth of the penetrating domain strip. The hollow arrows indicate the local orientation.

$10 \mathrm{~nm}$ [22]. Moreover, other MD simulations on BTO film of thickness less than $4.4 \mathrm{~nm}$ also showed the same domain size $[11,13]$. The weakening of retention can be attributed to the difference in the above-mentioned modes of domain switching and reorientations.

Furthermore, two models are devised to investigate the effect of i) pairs and triplets, which have moderate vacancy concentration, and ii) isolated vacancies, which have no interaction due to a longer separation distance, on the hysteresis loop. The former model is devised in such a manner that the ordered vacancies are located at the centre of a $6 \times 6 \times 25.5$ cells system and parallel to the \{101\} plane, containing 1, 2 and 3 vacancies denoted as monomer, dimer and trimer [8], respectively. Whereas, the latter model contains 2, 3 and 4 isolated vacancies uniformly distributed in the same system as the former. 
Consequently, the concentration in these two models is in the range of 0.04 to $0.14 \%$. The results obtained from the former model show an increase in residual polarization of $17.8 \%$, i.e., from $10.1 \pm 0.3$ to $11.9 \pm 0.2 \mu \mathrm{C} / \mathrm{cm}^{2}$, as the concentration is increased up to $0.11 \%$, in comparison with that of a film. And the coercive and saturated fields are greatly enhanced by $325 \%$, i.e., from 103 to $438 \mathrm{kV} / \mathrm{cm}$ and $71.4 \%$, i.e., from 700 to $1200 \mathrm{kV} / \mathrm{cm}$, respectively. The latter model also shows an increase in residual polarization of $9.9 \%$, i.e., from $10.1 \pm 0.3$ to $11.1 \pm 0.5 \mu \mathrm{C} / \mathrm{cm}^{2}$, as the concentration is increased up to the same level. However, the corresponding values of the coercive force are not monotonically increasing with the increase of concentration from 0.04 to $0.14 \%$, even though they are all greater than that of a film (note that the monomer can also be regarded as a case of isolated vacancy system with the lowest concentration). In addition, the trend of the saturated field is not very clear in this range. Refer to the details in table 1 . In short, both isolated vacancies and ordered vacancies would enhance the residual polarization and coercive force as compared with a film free of oxygen vacancies. The saturated field is also enhanced except for the monomer case. Moreover, the concentration of oxygen vacancy does not play a key role in the weakening of retention. On the contrary, a small amount of isolated oxygen vacancies can enhance the ferroelectric behavior.

The distributions of the magnitude and angle of polarization have been analyzed to investigate spontaneous polarizations for all the above-mentioned cases. No significant difference is observed in comparison with that of the $(6,4)$ array. The spontaneous polarization is mainly along [101] or the equivalent orientations.

In conclusion, an apparent weakening of retention is exhibited as the size of the oxygen vacancy ordering array in a $10 \mathrm{~nm}$ thick film is reduced to a critical level, in which decaying of residual polarization, coercive force and saturated field is illustrated. The array is parallel to the $\{101\}$ crystal plane and the critical size is characterized by the separation distance of 6 and 4 ordered cells along the [001] and [010] orientation, respectively. The weakening of retention is due to the variation of the domain switching mode. The zero retention arises from the formation and motion of head-to-head domain structure, which is different from the switching and reorientation mode of domains in a bigger array or a point defect-free film. It has also been found that a small amount of oxygen vacancy can enhance the ferroelectric behavior. It is important to note that the weakening of retention depends mainly on the size of the oxygen vacancy ordering array rather than on the vacancy concentration. The current work is valid for ultra-thin BTO films when the thermal perturbation and stress/strain effect are not taken into consideration. The study of thermal effect and spontaneous diffusion of oxygen vacancies from isolated to ordering or ordering array could be an interesting topic for future research.
The authors wish to thank Dr YiHUi ZHANG for helpful discussion. Support from the Research Grants Council of the Hong Kong Special Administrative Region, China (Project No. HKU 716007E), the NSFC (grant Nos. 10772012, 10732090, 10772181 and 10721202), the Chinese Academy of Sciences (CAS) Innovation Program (KJCX2YW-M04) and the NBRPC (2007CB814803) is gratefully acknowledged. Computations were performed on computer clusters at the CNIC Supercomputing Center, the LSEC of the CAS and the HPCPOWER system of computer centre of HKU.

\section{REFERENCES}

[1] Yun W. S., Urban J. J., Gu Q. et al., Nano Lett., 2 (2002) 447.

[2] Cheng B. L., Gabbay M., Maglione M. et al., J. Electroceram., 10 (2003) 5.

[3] Suzuki M., Kobayashi Y., Aoyagi S. et al., IEEJ Trans. Sens. Micromach., 129 (2009) 295.

[4] Guo Y., Akai D., Sawada K. et al., Solid State Commun., 149 (2009) 14.

[5] Salaoru I. and Paul S., Philos. Trans. A Math. Phys. Eng. Sci., 367 (2009) 4227.

[6] Chen Y., Liu B., Ma Y. et al., Nucl. Instrum. Methods Phys. Res. B, 267 (2009) 3090.

[7] Dawber M. and Scott J. F., Appl. Phys. Lett., 76 (2000) 1060.

[8] Scott J. F. and Dawber M., Appl. Phys. Lett., 76 (2000) 3801.

[9] Woodward D. I., Reaney I. M., Yang G. Y. et al., Appl. Phys. Lett., 84 (2004) 4650.

[10] Tinte S., Stachiotti M. G., Phillpot S. R. et al., J. Phys.: Condens. Matter, 16 (2004) 3495.

[11] Tinte S. and Stachiotti M. G., Phys. Rev. B, 64 (2001) 235403.

[12] Tinte S., Stachiotti M. G., Sepliarsky M. et al., J. Phys.: Condens. Matter, 11 (1999) 9679.

[13] Sang Y., Liu B. and Fang D., Comput. Mater. Sci., 44 (2008) 404.

[14] Zhang Y., Hong J., LiU B. et al., Nanotechnology, 20 (2009) 405703.

[15] Sepliarsky M., Asthagiri A., Phillpot S. R. et al., Curr. Opin. Solid State Mater. Sci., 9 (2005) 107.

[16] Mitchellt P. J. and Fircham D., J. Phys.: Condens Matter, 5 (1993) 1031.

[17] Wolf D., Keblinski P., Phillpot S. R. et al., J. Chem. Phys., 110 (1999) 8254.

[18] Smith W., Yong C. W. and Rodger P. M., Mol. Simul., 28 (2002) 385.

[19] McMichael R. D. and Donahue M. J., IEEE Trans. Magn., 33 (1997) 4167.

[20] Diéguez O., Tinte S., Antons A. et al., Phys. Rev. B, 69 (2004) 212101.

[21] Shin Y.-H., Grinberg I., Chen I.-W. et al., Nature, 449 (2007) 881.

[22] Drezner Y. and Berger S., J. Appl. Phys., 94 (2003) 6774 . 